A. Kather, J. B. Schulz, S. C. Tauber

Klinik für Neurologie, Universitätsklinikum RWTH Aachen, Aachen, Deutschland

\title{
Seltene Ursache einer Meningoenzephalomyelitis
}

\section{Anamnese}

Eine 48-jährige Patientin stellte sich in der internistischen Notaufnahme eines auswärtigen Krankenhauses aufgrund von seit 3 Tagen bestehendem Fieber, Kopfschmerzen sowie am Vorstellungstag neu hinzugekommenem Erbrechen vor. Die internistische sowie neurologische Untersuchung der als Bürokauffrau arbeitenden Patientin ohne relevante Vorerkrankungen war unauffällig. Bei fehlendem internistischem Infektfokus sowie normalen Routinelaborparametern (insbesondere normwertigen Leukozyten und normwertigem CRP [, c-reactive protein"]) erfolgte die Weiterbehandlung durch die Kollegen der dortigen Neurologie.

\section{Akuter klinischer Verlauf und Diagnosestellung}

Die in der externen Notaufnahme durchgeführte Liquoranalyse ergab eine lymphomonozytäre Pleozytose von $160 / \mu$ l bei deutlicher Eiweißerhöhung, normaler Glukose und minimaler Laktaterhöhung. Eine empirische Behandlung mittels Ceftriaxon und Aciclovir wurde umgehend eingeleitet und die antivirale Therapie mit Aciclovir nach Erhalt negativer HSV(Herpes simplex Virus)- und VZV(Varizella zoster Virus)-PCR („polymerase chain reaction") wieder beendet. Nach subjektiver Besserung des Allgemeinbefindens der bis dato klinisch-neurologisch unauffälligen Patientin kam es am insgesamt 5. Tag der Behandlung zu Doppelbildern. Eine erneute Liquoranalyse zeigte eine

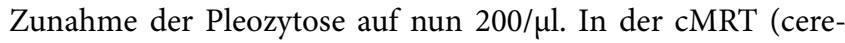
brale Magnetresonanztomographie) fand sich eine basale leptomeningeale Kontrastmittelaufnahme. Eine tuberkulostatische Therapie mit Isoniazid, Pyrazinamid, Ethambutol und Streptomycin wurde begonnen. Eine Röntgenuntersuchung des Thorax sowie eine Abdomensonographie ergaben keinen relevanten Befund.

Die Patientin entwickelte am Folgetag eine psychomotorische Verlangsamung mit Desorientiertheit und Dysarthrie. Unter der Verdachtsdiagnose einer tuberkulösen Meningitis mit klinischer Verschlechterung unter 1-tägiger empirischer Behandlung erfolgte 10 Tage nach Auftreten der ersten Symptome die Verlegung zu uns auf die Intensivstation der Klinik für Neurologie des Universitätsklinikums Aachen. Im neurologischen Untersuchungsbefund präsentierte sich die Patientin zu diesem Zeitpunkt somnolent mit reduzierter Sprachproduktion und geringen Spontanbewegungen aller Extremitäten.

\section{Redaktion}

A. Münchau, Lübeck

W. Pfeilschifter, Frankfurt am Main

S. Schmidt, Bonn

DGNeurologie $2020 \cdot 3$ (3): 234-237

https://doi.org/10.1007/s42451-020-00166-w

Online publiziert: 25 . Februar 2020

(c) Der/die Autor(en) 2020
Bei der erneuten Liquorentnahme zeigten sich ein erhöhter Liquoreröffnungsdruck von $37 \mathrm{~cm} \mathrm{H}_{2} \mathrm{O}$ und eine Pleozytose von $89 / \mu \mathrm{l}$ mit deutlich gestiegener Eiweißerhöhung auf 3,9g/l, Erniedrigung der Glukose auf $30 \mathrm{mg} / \mathrm{dl}$ sowie einer Laktaterhöhung auf $4,2 \mathrm{mmol} / \mathrm{l}$.

\section{॥ Liquoranalytische und bildmorphologische Verdachtsdiagnose: Tuberkulöse Meningitis}

Bei respiratorischer Erschöpfung erfolgten noch am Tag der Übernahme die orotracheale Intubation und bei fortgesetzter Beatmungspflichtigkeit im Verlauf eine Tracheotomie.

In der cMRT ließ sich weiterhin eine basal betonte leptomeningeale Kontrastmittelaufnahme nachweisen, zusätzlich fanden sich eine Diffusionsstörung des Balkens und in der spinalen MRT eine in der Medulla oblongata beginnende dorsale Myelitis (Abb. 1a-c).

Nachdem die Erregerdiagnostik insbesondere hinsichtlich Mykobakterien negativ verlief und sich die klinische Symptomatik nicht besserte, wurde die antibiotische Therapie beendet. $\mathrm{Da}$ im Liquor positive oligoklonale Banden nachgewiesen worden waren, wurden eine autoimmunologische Diagnostik des Serums und Liquors veranlasst und die Patientin bereits vor Erhalt der Ergebnisse mittels hochdosierter Kortisonstoßtherapie (Prednisolon $1 \mathrm{~g}$ i.v./Tag) behandelt.

Es konnten am Institut für Klinische Chemie des Universitätsklinikums Schleswig-Holstein mittels Gewebeassay (indirekte Immunfluoreszenz) positive Anti-GFAP-Antikörper (GFAP: "glial fibrillary acidic protein") in Serum und Liquor $(1: 100)$ nachgewiesen werden, sodass wir die Diagnose einer autoimmunen GFAP-Astrozytopathie stellten. Anti-Aquaporin-4- sowie Anti-MOG(„myelin oligodendrocyte protein“)Antikörper waren negativ.

\section{I) Diagnose: Autoimmune GFAP-Astrozytopa- thie}

Der weitere klinische Verlauf zeigte unter fortgeführter Kortisontherapie eine stetige Regredienz der klinischen Symptomatik, sodass ein erfolgreiches Weaning und eine Dekanülierung erfolgten. Nachdem die Patientin initial eine spastische Tetraplegie aufgewiesen hatte, war sie im weiteren Verlauf orientiert und zeigte zunehmende Willkürbewegungen von Armen und Beinen. In der augenärztlichen Mitbeurteilung fand sich eine im Verlauf rückläufige (Stauungs-)Papillitis.

In der Verlaufsliquoranalyse fanden sich jetzt ein normaler Liquoreröffnungsdruck, eine Normalisierung des Liquorei-

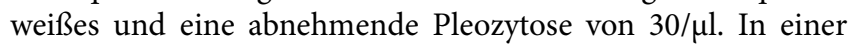
Verlaufs-MRT nach 9 Tagen war die Diffusionsrestriktion im Bereich des Balkens rückläufig (Abb. 1d).

Die Patientin trat unter einer oralen Prednisolontherapie von $40 \mathrm{mg} /$ Tag eine neurologische Rehabilitation an. 
Hier steht eine Anzeige.

Springer 

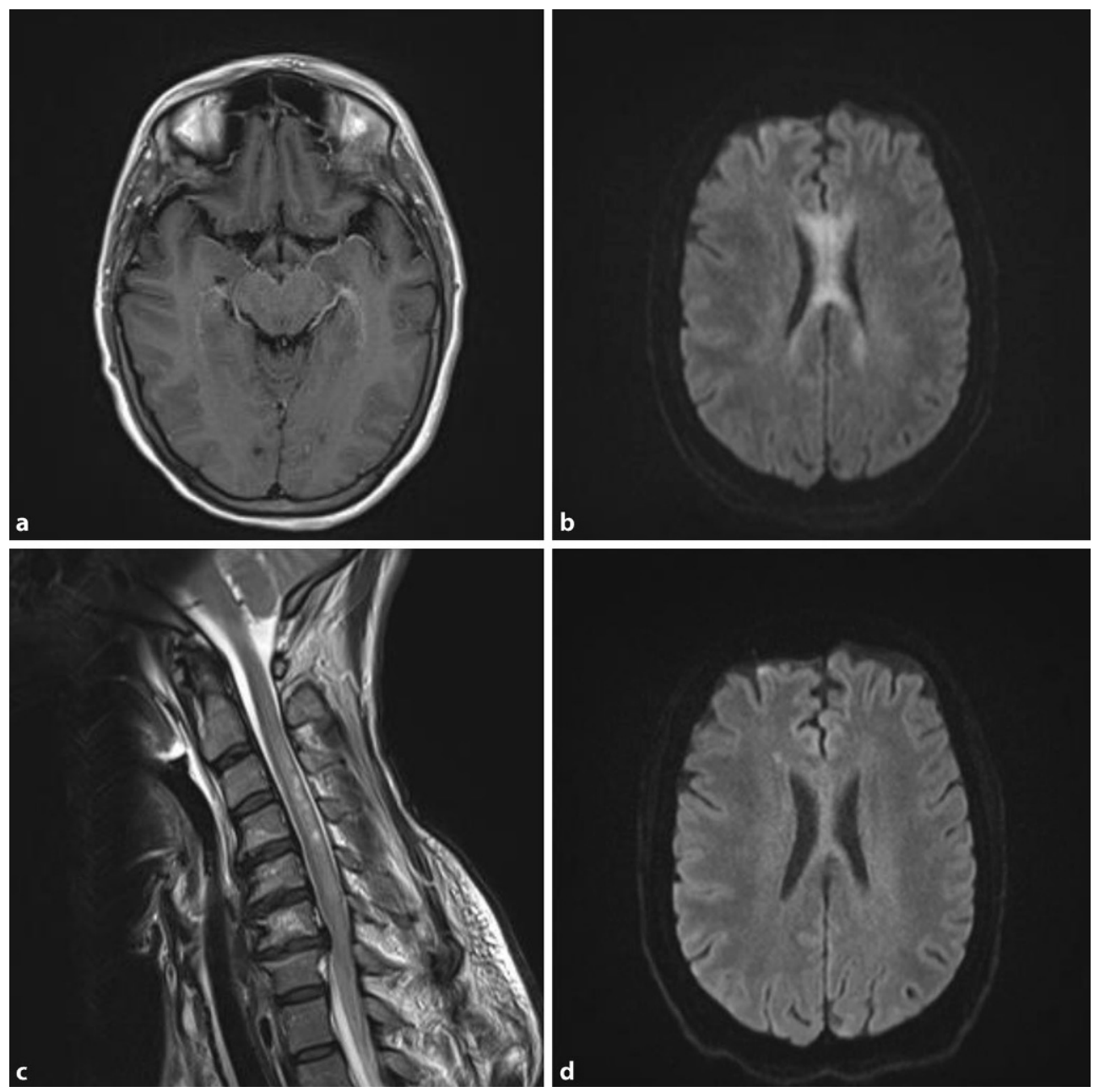

Abb. 1 Zerebrale und spinale MRT im Verlauf: a zerebrale MRT mit leptomengingealem Enhancement der zerebralen Zisternen (T1_tirm-Blade KM tra_dark-fluid), $\mathbf{b}$ zerebrale MRT mit Diffusionsstörung des Balkens (ep2d_diff_3scan_trace_p2 TRACEW), c spinale MRT mit T2-hyperdenser fleckförmiger Läsion (T2_tse_sag_HWS), d Verlaufs-MRT nach 9 Tagen unter Kortisonstoß mit regredienter Diffusionsstörung des Balkens

\section{Poststationärer Verlauf und weitere Diagnostik}

Nach 3 Monaten erfolgte eine ambulante Verlaufskontrolle in unserer neuroimmunologischen Ambulanz. Im neurologischen Untersuchungsbefund zeigte sich ein spastisch-ataktisches Gangbild, eine neuropsychologische Testung ergab einen Normalbefund, die externe Kontroll-cMRT eine nahezu unauffällige Darstellung (Abb. 1c), ein GFAP-Titer im Serum war aber weiterhin nachweisbar (1:320). Die verbliebene Prednisolondosis wurde schrittweise ausgeschlichen. Erneute Kontrollen nach 6 und 12 Monaten ergaben keine neuen klinischen Symptome.

Eine Malignomsuche inklusive einer Ganzkörper-PETCT (Positronen-Emissions-Computertomographie) war bereits zum Zeitpunkt des stationären Aufenthaltes mit unauffälligem Ergebnis erfolgt. Eine erneute PET-CT nach 6 Monaten ergab weiterhin keinen Hinweis auf ein Malignom.

Nach knapp einem Jahr klinischer Verlaufskontrollen ergaben sich keine Hinweise auf ein Rezidiv.

\section{Diskussion}

Durch die wachsenden Kenntnisse über und die Nachweisbarkeit von Autoimmunantikörpern ist die Zahl klinisch definierter und serologisch nachweisbarer Autoimmunenzephalitiden als Ursache neurologisch-psychiatrischer Syndromkomplexe erheblich gestiegen [1,2]. Mit dem vorliegenden Fall, bei dem zunächst aufgrund des Liquor- und bildmorphologischen
Befundes eine tuberkulöse Meningitis vermutet wurde, möchten wir eine erst kürzlich entdeckte und seltene Ursache einer Meningoenzephalomyelitis präsentieren.

Die Anti-GFAP-Astrozytopathie wurde erstmals 2016 beschrieben [3]. Von den in der Literatur aufgearbeiteten Fällen zeigten sich die meisten als Voll- oder Teilbild einer Meningoenzephalomyelitis [2-4], wie auch im vorliegenden Fallbericht. Als initiale Symptome wurden in der Literatur Fieber, Kopfschmerzen sowie vorausgehende grippeähnliche Symptome als häufigste primäre Manifestation beschrieben [2,3]. Die initiale rapide Verschlechterung, die unsere Patientin erlebte, ist ebenso typisch wie die Liquorveränderungen [2,3] und die MR-morphologische Präsentation [2,3]. Die Papillitis ist ebenfalls Teil der Erkrankung, als deren Ursache nicht nur eine intrakraniale Hypertension, sondern auch direkte entzündliche Veränderungen am N. opticus vermutet werden [3]. Im vorliegenden Fall nicht nachweisbare, aber in der Literatur beschriebene Symptome einer Anti-GFAP-Astrozytopathie sind Halluzinationen und Bewegungsstörungen meist im Sinne einer Ataxie sowie autonome Funktionsstörungen [3].

Der Nachweis von Anti-GFAP-IgG im Liquor ist spezifischer als der serologische Nachweis [2]. Als Bestandteil des Intermediärfilaments der Astrozyten ist GFAP ein intrazelluläres Protein. Die Relevanz dieses Proteins wurde bereits beim M. Alexander erforscht, einer Leukodystrophie, die durch eine 
De-novo- oder autosomal dominante Mutation des GFAP kodierenden Gens bei Kindern, aber auch im Erwachsenenalter auftritt [5].

Ätiologisch wird aufgrund der intrazellulären Lokalisation des GFAP bei der autoimmunen Astrozytopathie ein T-Zellvermittelter Mechanismus angenommen [3]. Dieser entsteht bei einem Teil der Patienten wahrscheinlich durch molekulares Mimikry, da bei ungefähr 1/3 der Anti-GFAP-Astrozytopathien ein Malignom gefunden wurde [2, 3, 6]. Sekundäre Astrozytopathien nach infektiöser VZV- oder HSV-Enzephalitis wurden ebenfalls beschrieben [2].

Die anatomische Verteilung der bei der autoimmunen GFAP-Astrozytopathie nachgewiesenen Areale in der MRT ist ähnlich der der Neuromyelitis-optica-Spektrum-Erkrankungen (NMOSD; [7]). Bei NMOSD richten sich Antikörper u.a. gegen das Protein Aquaporin-4, welches sich auf der Oberfläche der Astrozyten befindet. Die in der Literatur bei autoimmuner GFAP-Astrozytopathie beschriebenen T2-hyperintensen und/ oder kontrastmittelaufnehmenden Bereiche finden sich u. a. periventrikulär, leptomeningeal sowie spinal [4]. In einer Studie wurde in $83 \%$ der Patienten ein radiäres Muster des periventrikulären Enhancements als sehr charakteristisch beobachtet [7]. In unserem Fall ist die regrediente Diffusionsstörung des Corpus callosum besonders erwähnenswert, da diese in der Literatur im Kontext der autoimmunen GFAP-Astrozytopathie noch nicht beschrieben wurde.

Die Symptome sprechen in der Mehrzahl der Fälle gut auf Steroide an. Rezidive wurden in rund $20 \%$ der Fälle beobachtet, ein fehlendes Ansprechen auf die initiale Therapie weist auf einen zugrunde liegenden Tumor und/oder das Vorliegen weiterer Autoimmunantikörper, wie Anti-Aquaporin-4- oder Anti-NMDA(N-Methyl-D-Aspartat)-Rezeptor-Antikörper, hin [2]. Neben der Behandlung möglicher maligner Grunderkrankungen ist bei Rezidiven der längerfristige Einsatz steroidsparender Immunsuppressiva beschrieben [2].

Zusammenfassend stellt die autoimmune Anti-GFAP-Astrozytopathie eine erst seit kurzem bekannte Form der autoimmunen Meningoenzephalitiden dar, die trotz ihrer Seltenheit in die differentialdiagnostischen Überlegungen aufgenommen werden sollte und mittels Liquoranalyse zu diagnostizieren und mittels Steroiden gut therapierbar ist.

\section{Fazit für die Praxis}

- Als Differenzialdiagnose der nichtinfektiösen Meningoenzephalomyelitis sollte die GFAP(„glial fibrillary acidic protein“)Astrozytopathie in Erwägung gezogen werden.

- Der Nachweis von Anti-GFAP-Immunglobulin-G sollte aus dem Liquor erfolgen.

- Es finden sich entzündliche Liquor- und MRT(Magnetresonanztomographie)-Veränderungen.

- Ein gutes und schnelles Ansprechen auf Steroide ist typisch.

- Eine Malignomsuche sollte - insbesondere bei ausbleibendem Therapieerfolg - gründlich erfolgen.

Literatur

1. Ramanathan S, Al-Diwani A, Waters P, Irani S (2019) The autoantibody-mediated encephalitides: from clinical observations to molecular pathogenesis. J Neurol

https://doi.org/10.1007/s00415-019-09590-9
2. Dubey D, Hinson S, Jolliffe E, Zekeridou A, Flanagan E, Pittock S, Basal E, Drubach D, Lachance D, Lennon V, McKeon A (2018) Autoimmune GFAP astrocytopathy: prospective evaluation of 90 patients in 1 year. J Neuroimmunol 321:157-163 3. Fang B, McKeon A, Hinson S, Kryzer T, Pittock S, Aksamit A, Lennon V (2016) Autoimmune glial fibrillary acidic protein astrocytopathy. JAMA Neurol 73:1297

4. Kunchok A, Zekeridou A, McKeon A (2019) Autoimmune glial fibrillary acidic protein astrocytopathy. Curr Opin Neurol 32:452-458

5. Johnson A (2004) Alexander disease: a leukodystrophy caused by a mutation in GFAP. Neurochem Res 29:961-964

6. Flanagan E, Hinson S, Lennon V, Fang B, Aksamit A, Morris P, Basal E, Honorat J, Alfugham N, Linnoila J, Weinshenker B, Pittock S, McKeon A (2017) Glial fibrillary acidic protein immunoglobulin $G$ as biomarker of autoimmune astrocytopathy: analysis of 102 patients. Ann Neurol 81:298-309

7. Sechi E, Morris P, McKeon A, Pittock S, Hinson S, Weinshenker B, Aksamit A, Krecke K, Kaufmann T, Jolliffe E, Zalewski N, Zekeridou A, Wingerchuk D, Jitprapaikulsan J, Flanagan E (2018) Glial fibrillary acidic protein IgG related myelitis: characterisation and comparison with aquaporin-4-lgG myelitis. J Neurol Neurosurg Psychiatry 90:488-490

\section{Korrespondenzadresse}

\section{A. Kather}

Klinik für Neurologie

Universitätsklinikum RWTH Aachen

Pauwelsstraße 30, 52074 Aachen, Deutschland

akather@ukaachen.de

\section{Einhaltung ethischer Richtlinien}

Interessenkonflikt. J.B. Schulz gibt folgende Interessenkonflikte an: Scientific advisory board Novartis, MSD; Reise- und Vortragshonorare von Pfizer, Novartis, Bayer; Editor in chief des Journal of Neurochemistry; Editorial board für Synapse. S.C. Tauber gibt folgende Interessenkonflikte an: Scientific advisory board Roche; Reise- und Vortragshonorare von Novartis, Tena, Merck, Roche und Biogen. A. Kather gibt an, dass kein Interessenkonflikt besteht.

Für diesen Beitrag wurden von den Autoren keine Studien an Menschen oder Tieren durchgeführt. Für die aufgeführten Studien gelten die jeweils dort angegebenen ethischen Richtlinien. Für Bildmaterial oder anderweitige Angaben innerhalb des Manuskripts, über die Patienten zu identifizieren sind, liegt von ihnen und/oder ihren gesetzlichen Vertretern eine schriftliche Einwilligung vor.

Open Access. Dieser Artikel wird unter der Creative Commons Namensnennung 4.0 International Lizenz veröffentlicht, welche die Nutzung, Vervielfältigung, Bearbeitung, Verbreitung und Wiedergabe in jeglichem Medium und Format erlaubt, sofern Sie den/die ursprünglichen Autor(en) und die Quelle ordnungsgemäß nennen, einen Link zur Creative Commons Lizenz beifügen und angeben, ob Änderungen vorgenommen wurden.

Die in diesem Artikel enthaltenen Bilder und sonstiges Drittmaterial unterliegen ebenfalls der genannten Creative Commons Lizenz, sofern sich aus der Abbildungslegende nichts anderes ergibt. Sofern das betreffende Material nicht unter der genannten Creative Commons Lizenz steht und die betreffende Handlung nicht nach gesetzlichen Vorschriften erlaubt ist, ist für die oben aufgeführten Weiterverwendungen des Materials die Einwilligung des jeweiligen Rechteinhabers einzuholen.

Weitere Details zur Lizenz entnehmen Sie bitte der Lizenzinformation auf http://creativecommons.org/licenses/by/4.0/deed.de.

Funding. Open Access funding provided by Projekt DEAL. 